\title{
A Standardized Plant Extract Containing a Target Compound is Acceptable as a Potent Therapeutic Entity: Relevance to BEC and Solamargine, a Topical Clinical Formulation Curaderm
}

\author{
Aruba Cham ${ }^{1}$, Kai Cham², Tania Chase ${ }^{2}$, Bill Cham ${ }^{1}$ \\ ${ }^{1}$ Department of Oncology, Australasian Medical Research, Port Vila, Republic of Vanuatu \\ ${ }^{2}$ Research Section, Curaderm Global, Port Vila, Republic of Vanuatu
}

\section{Email address:}

bill.cham@gmail.com (B. Cham)

\section{To cite this article:}

Aruba Cham, Kai Cham, Tania Chase, Bill Cham. A Standardized Plant Extract Containing a Target Compound is Acceptable as a Potent Therapeutic Entity: Relevance to BEC and Solamargine, a Topical Clinical Formulation Curaderm ${ }^{\text {BEC5 }}$. Journal of Cancer Treatment and Research. Vol. 3, No. 2, 2015, pp. 22-27. doi: 10.11648/j.jctr.20150302.12

\begin{abstract}
New approaches to curb developmental and end-stage production expenses, whilst generating a safe and effective treatment for cancer, resulting in lower cost for the patient and healthcare system, are warranted. A bioassay-guided fractionation of a Solanum plant, S. sodomaeum, to a standardized extract can be classified as a new drug. It is shown that a specific standardized plant extract, BEC, containing a given concentration of a target efficacious and safe compound, solamargine, is an acceptable anticancer and less costly chemotherapeutic reality.
\end{abstract}

Keywords: Cancer, Solamargine, BEC, Curaderm, Solanum, Plant Extract

\section{Introduction}

New drug development is a complex, time consuming and expensive process. Over 12 years are required to take drug discovery of a new drug to clinical application. In today's context this involves more than 1 billion US\$ of investment [1]. Traditional anticancer drug therapies are not only costly, but most concerning, these therapies are nonselective and very toxic resulting in a lack of satisfactory results in cancer treatment. Oncologists have now recognized this and have resorted to combinational anticancer therapy utilizing multiple traditional antineoplastic agents. Still the results of these therapies are not fulfilling the needs and expectations of patients and health care professionals.

Due to the alarming lack of safe, effective treatments for cancer, considerable interests have been drawn to naturally occurring smart drugs. More than $80 \%$ of drug substances are purely natural products or were inspired by molecules derived from natural sources. Smart drugs are specific toxins that focus on a particular vulnerability of the cancer, they are specific toxins. In particular, the glycoalkaloids solasodine rhamnosides have attracted much attention. Glycoalkaloids are found in over hundreds of Solanum species. These
Solanum glycoalkaloids differ in their glycosidic as well as in their alkaloidal components. Different Solanum plants contain various amounts of percentages of such components. In nature glycoalkaloids are produced in plants as secondary metabolites and are not involved in metabolic activity. They act as defense chemicals. Their absence does not cause ill effects in the plants. However, in plants, glycoalkaloids are used to fight off pathogens such as bacteria, fungi, viruses, worms and humans, they are considered to be defensive allelo-chemicals [2-4].

Cancer is cruel to the human body and a cruel environment is cancerous to plants. Plants, which are able to comfortably survive under multiple but diverse hostile environments, may be associated with a vital source of anticancer agents.

Solanum plants, through evolution, have produced solasodine rhamnosides allelo-chemicals. This has resulted in their survival under multiple and diverse hostile environments such as being exposed to a number of detrimental pathogens. Subsequently these solasodine rhamnosides have shown to be potent anticancer agents at different stages of cancer development. They cause cancer cells to commit suicide (apoptosis) [5-31], are cytotoxic against multi drug resistant tumour cells $[7,14,31]$, stimulate 
lasting immunity against cancer $[9,31]$, are not mutagenic and even display anti mutagenic properties [7, 31].

In 1987 it was first reported that an Australian local Solanum species, Devil's Apple, Solanum sodomaeum (linnaeanum) possessed anticancer properties [5-7]. Several active ingredients, including solamargine, solasonine, monoglycosides and diglycosides of solasodine were reported to express varying degrees of anticancer activities in the $S$. sodomaeum extracts. Analyses of the extract indicated that these solasodine glycosides were present in a specific standardized mixture known as $\operatorname{BEC}[5,8,9]$. BEC is composed of solamargine $(33 \%)$, solasonine $(33 \%)$ and di- and monoglycosides of solasodine (34\%) [5-9].

Solasodine glycosides in BEC have a mono or oligosaccharide chain attached to the $\mathrm{C} 3$ position of the nitrogenous steroid alkaloid solasodine backbone. The triglycoside solamargine contains two molecules of rhamnose, and the triglycoside solasonine contains one molecule of rhamnose. Depending on the diglycosides present in BEC, they may contain one or no rhamnose molecule, whereas the monoglycoside in BEC contains no rhamnose molecule.

Figure 1 shows the chemical structures of solasonine and solamargine.

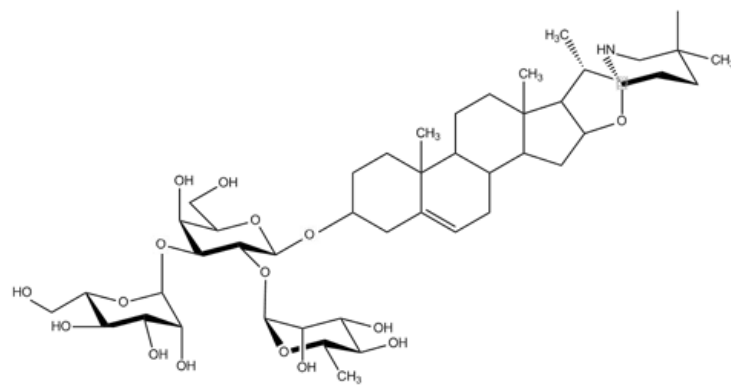

Solasonine

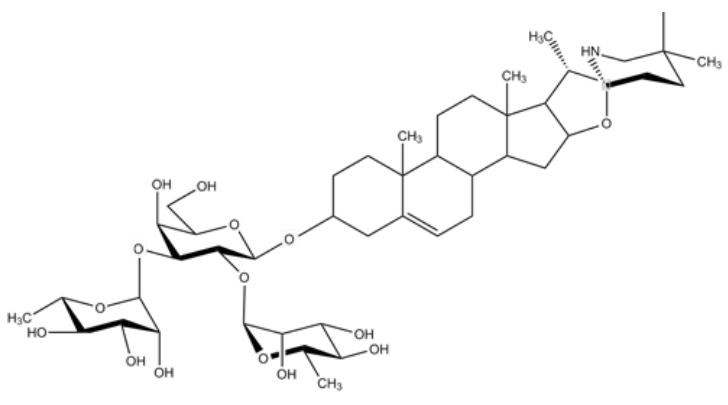

Solamargine

Figure 1. Chemical structures of solasonine and solamargine

The structure-activity relationship of these solasodine glycosides has been reported. The anticancer properties of solamargine, solasonine and their hydrolysis products beta 1 , beta 2 , and gamma solamargine and beta 2 and gamma solasonine and their aglycone solasodine revealed that solamargine and solasonine were the most active [10]. The carbohydrate side chains of these glycoalkaloids are paramount in influencing biological activity. Not only the number but also the type of carbohydrate, as well as the order of attachment affect the anticancer activity of the glycoalkaloids [10].

The two rhamnose molecules moieties of solamargine play a crucial role in triggering cancer cell death by apoptosis. The trisaccharide of solamargine, in which two rhamnose units are connected to a glucose moiety, binds more efficiently to specific cell receptor sites than the corresponding trisaccharide solasonine in which one rhamnose and oneglucose units are bridged by a galactose monosaccharide. A specific cell receptor has been identified as an endogenous endocytic lectin [8] and has been characterised as a rhamnose binding protein on the cancer cell membrane $[11,12]$.

Solasodine rhamnosides in BEC are now considered to be the much-sought class of targeted cancer therapy and can be classified as smart drugs. After rhamnose binding to the specific receptors, the entire solasodine rhamnosides are internalized by receptor-mediated endocytosis through "coated pit endocytosis" and a receptorsome or endosome is formed. Once internalized in the cancer cell the solasodine moiety then expresses its importance in antineoplastic activities, and, the lysosomes and mitochondria are affected.
Consequently, extrinsic and intrinsic apoptotic pathways in the cancer cell are triggered resulting in up-regulation of the expressions of external death receptors, such as tumour necrosis factor receptors (TNFR-1 and TNFR-2), Fas receptor, TNFR-1 associated death domain and Fasassociated death domain. The solasodine rhamnosides enhance the intrinsic ratio of $\mathrm{Bax}$ to $\mathrm{Bcl}-2$ by up-regulating $\mathrm{Bax}$ and down-regulating $\mathrm{Bcl}-2$ and $\mathrm{Bcl}-\mathrm{xL}$ expressions. These effects result in activation of Caspase $-8,-9$ and -3 in cancer cells, a hallmark of apoptosis, which was confirmed by morphological studies $[8,13]$.

Apoptosis, or programmed cell death, is a naturally occurring process in the body. Healthy cells eliminate themselves by apoptosis when they become defective. Cancer cells resist apoptosis and continue to multiply uncontrollably, resulting in an increased tumour mass. Resisting apoptosis is a key process in cancer formation and proliferation. BEC specifically induces apoptosis in cancer cells making it a powerful therapeutic tool $[7,8,14]$.

Studies with BEC, its individual and combinational individual components consisting of triglycosides solamargine, solasonine, diglycosides and monoglycosides of solasodine clearly show that these solasodine rhamnosides are far superior to existing chemotherapeutic agents $[7,15-$ 17]. A topical cream formulation, Curaderm ${ }^{\mathrm{BEC} 5}$, containing BEC is currently used clinically for the treatment of skin cancers [7, 18-25].

A clear distinction in terms of efficacy, safety and cost have hitherto not been reported in a clinical setting for a target potent anticancer compound when this compound is 
present in a standardized extract containing other less effective antineoplastic agents.

In this report it is shown that under specified conditions, BEC, with known contents of solamargine, can be used clinically for various diseased states. This translates to reducing the costs of cancer treatment for the health system and for those affected by cancer. This is achieved by reducing the costs of producing the anticancer preparation since it is much more expensive to obtain isolated purified solamargine than it is to obtain the BEC extract.

\section{Results}

\subsection{Cytotoxic Activities of BEC and Its Individual Solasodine Glycosides}

Daunter and Cham reported that the relative cytotoxic activities of BEC against various cancer cell lines were mainly due to solamargine, whereas solasonine and the mono-and-diglycosides of solasodine had limited cytotoxic activities relative to solamargine [6-8]. Using a variety of cancer and normal cell lines it was shown that solamargine was 15 times more effective than solasonine and 13 times more effective than a mixture of diglycosides and monoglycosides of solasodine [7, 26].

Carter et al. reported that the cytotoxicity of purified solamargine (SBP04) against the human cell lines mesothelioma (NO36), prostate adenocarcinoma (LNCaP), colonic carcinoma (LS174T), melanoma (A2058) and renal cell carcinoma (786-0) was more than 4 times higher than that of purified solasonine (SBP05). Furthermore, solamargine-quantified $\mathrm{BEC}$ with measured $\mathrm{IC}_{50}$ in various human cancer cell lines were similar to that of pure solamargine [27].
Cui et al. have synthesized various solasodine glycosides and evaluated and compared their cytotoxic effects with solamargine on human cancer cells. They showed that the cytotoxicity of solamargine on four human cancer cell lines, squamous cell carcinoma (KB), breast adenocarcinoma (MCF-7), myelogenous leukemia (K562) and prostate cancer (PC3) was much higher than 9 variations of synthesised solasodine glycosides, including the monoglycoside solasodine rhamnoside. The $\mathrm{IC}_{50}$ of solamargine for human cancer cell lines was more efficient than other solasodine glycosides by factors ranging from 2.7 to 8.9 [28].

$\mathrm{Wu}$ et al. showed that the cytotoxicity of solamargine against squamous cell carcinoma (SCC25) was 17 times higher than that of solasonine. Furthermore, solamarginequantified $S$. incanum extract SR-T100 (an extract which is similar to BEC) had identical efficacy compared with pure solamargine [29].

Shabana et al. studied the anticancer activity of an extract MEP (another extract which is similar to BEC) and its individual glycoalkaloids of the fruit peels of $S$. melongena and demonstrated that solamargine was 2.38 more potent than solasonine in antineoplastic activity in 5 human cancer cell lines - colon cancer (HCT116), larynx cancer (HEP2), breast cancer (MCF7), cervix cancer (HELA) and liver cancer (HEPG2) [30].

Table 1 shows the relative anticancer activities of various solasodine conjugates against a large range and number of cancers. Relative, and for comparison of activities, the most effective substance is rated as $100 \%$. In all cases this was solamargine. On average solamargine is 9 and 19 times more effective against cancer than solasonine and mono-anddiglycosides of solasodine respectively.

Table 1. Relative Percent Anticancer Activities

\begin{tabular}{lllllll}
\hline & Compound BEC & Compound BEC & Compound SR-T100 & Compound MEP & Synthesized & SBP04, SBP05 \\
\hline Solamargine & 100 & 100 & 100 & 100 & 100 & 100 \\
Solasonine & 13 & 7 & 6 & 42 & - & 25 \\
Di, monoglycosides & 10 & 8 & $<2$ & - & 20 & - \\
Reference & 8 & 26 & 29 & 30 & 28 & 27 \\
\hline
\end{tabular}

Compounds BEC, SR-T100, MEP are different extracts containing a mixture of solamargine, solasonine, diglycosides of solasodine and monoglycosides of solasodine. Synthesized denotes synthesized solamargine and other variations, including monoglycosides, of solasodine. SBP04 and SBP05 are extracted purified solamargine and solasonine respectively.

\subsection{Toxicities of Solamargine and BEC in Humans}

Table 2 demonstrates concentrations of BEC and solamargine used in humans. Large concentration ranges of solamargine $(0.02-166 \mathrm{mg} / \mathrm{mL})$ and BEC $(0.05-500 \mathrm{mg} / \mathrm{mL})$ have been used safely in humans. Toxicity studies in animal models have been previously reported [7].

Table 2. Doses of Solamargine used in Humans

\begin{tabular}{|c|c|c|c|c|}
\hline Solamargine Concentration $\mathrm{mg} / \mathrm{mL}$ & Parent Compound & Administration & Phase Clinical Study & Reference \\
\hline $7-166$ & $\mathrm{BEC}$ & Topical & I & 4,31 \\
\hline 33 & $\mathrm{BEC}$ & Topical - BEC02 & II & 4,31 \\
\hline 0.02 & $\mathrm{BEC}$ & Topical - Curaderm & III & 4,31 \\
\hline 0.02 & Solamargine & Topical & Pilot clinical & 32 \\
\hline 23 & SR-T100 & Topical & Pilot clinical & 29 \\
\hline 0.03 & $\mathrm{BEC}$ & Intralesion & Pilot clinical & 4,31 \\
\hline 2 & $\mathrm{BEC}$ & Oral & Pilot clinical & 4,31 \\
\hline
\end{tabular}


A wide concentration range of solamargine in various extracts and in isolated purified form have been used clinically in human Pivotal Clinical Studies and Phases I to III Clinical Trials.

\section{Discussion}

The cost for bringing a new cancer drug to market is reported to be about US\$1 billion [1]. The cost includes development of the new (successful) drug and all other drugs that failed during development, conducting the clinical trials required for approval, bonuses, salaries, infrastructures, and advertising amongst others.

However, the justification of the claim that about US\$1 billion in costs to bring a new cancer drug to market and consequently the high price of the drug charged to the patient are hotly disputed [33]. Anticancer drugs rank first of global spending by therapeutic class. New anticancer drugs are more expensive than older products. The cost of the new generation of drugs is getting out of all proportion to the added benefit and is becoming unsustainable. For example, the newly approved melanoma drug ipilinumab costs US $\$ 120,000$ for a course of therapy. The drug was shown to be associated with an incremental increase in life expectancy of four months! This suggests that increases in prices of anticancer drugs are unrelated to real health improvements. Of the 12 anticancer drugs approved by the FDA in 2012, 11 were priced above US $\$ 100,000$ per year. This represents a doubling of prices from a decade ago. This level of expense is simply unacceptable since $1 / 3$ of people will get cancer in their lifetime. Dealing with burgeoning costs of cancer treatment is a major challenge for the health system and for those affected by cancer.

Multiple factors are involved in cancer drug pricing and impact on individual patients and healthcare policies. The need to lower the prices of cancer drugs to allow more patients to afford them and to maintain sound long-term healthcare policies are warranted.

One such factor is the production cost of the anticancer drug for its use in clinical settings.

Solamargine is a suitable glycoalkaloid with excellent antineoplastic properties both in terms of specificity and safety [7]. Solamargine is found in a large number of plant Solanum species. However, to date no Solanum plants have been found to contain solamargine on its own. In nature, solamargine is found in plant extracts, which also contain solamargine-related biomolecules such as solasonine, monoand diglycosides of solasodine and unbound solasodine.

The compound BEC is an extract that contains the above glycosylated solasodines. In particular, BEC consists of a constant mixture of solamargine, solasonine and di- and mono-glycosides of solasodine. The triglycosides solamargine and solasonine as well as the mono-and diglycosides of solasodine possess antineoplastic properties with differing activities.

At equimolar concentrations, on average, solamargine is 9 times more efficacious than solasonine and 19 times more efficacious than the mono-and-diglycosides of solasodine. BEC consists of $1 / 3$ solamargine, $1 / 3$ solasonine and $1 / 3$ mono-and-diglycosides of solasodine [5]. When calculated, the contribution of anticancer activities in BEC is $86 \%$ for solamargine, $9 \%$ for solasonine and $5 \%$ for mono-and diglycosides of solasodine. Solamargine-quantified BEC with measured $\mathrm{IC}_{50}$ values in various human cancer cell lines are similar to that of pure solamargine. This suggests that there are no synergistic effects of solamargine with the other solasodine glycosides found in BEC and that solamargine is the most effective anticancer component in BEC.

These observations have been elaborated in a clinical setting when treating human basal cell carcinomas. It was shown that topical cream formulations with identical excipients containing $0.02 \mathrm{mg}$ purified isolated solamargine per $1 \mathrm{~mL}$ cream had comparable therapeutic outcomes as $0.05 \mathrm{mg}$ BEC (containing $0.02 \mathrm{mg}$ solamargine) per $1 \mathrm{~mL}$ cream [32]. The concentrations of BEC and solamargine for effective skin cancer treatment are similar to those reported in cell culture studies for a wide variety of cancer cells.

The extraction of BEC from a wide variety of Solanum species is relatively not very costly nor time consuming. However, the isolation of the individual solasodine rhamnosides including solamargine from plant material or from BEC is very time consuming and costly.

There are challenges that have to be overcome when seeking ways of reducing the costs of cancer treatment for the health system and for those affected by cancer. A particular expense that contributes to the pricing of such treatments is the cost of producing the anticancer preparations.

In assessing such a challenge, there are many factors to consider. Two such main factors are safety and efficacy. Both these factors for BEC have been addressed in this article.

Reducing the production cost of an anticancer drug consequently should result in a significant cost lowering in the final product for treating this disease. In the United States alone treatment of skin cancer amounts to US\$1.8 billion each year [34]. Any modality that can reduce such a financial burden to the Health Care System and to patients should be considered seriously.

In the case of Curaderm, a clinically available topical treatment for skin cancer, it has been established that BEC with a quantifiable known amount of solamargine is an acceptable preparation for its indications [6-9, 16-27, 29, 31, 32, 35-43].

This paper shows that it is possible to reduce the costs associated with the production of treatment preparations by using well-characterized extracts containing known amounts of the principal anticancer ingredient.

Currently, further work is being undertaken to establish the clinical relevance of BEC for other more serious cancers. 


\section{References}

[1] Goozer, M. (2004) The $\$ 800$ million pill: The truth behind the cost of new drugs. University of California Press. Berkley, CA.

[2] Chataing, B., Christancho, N.B. and Usubillaga, A. (1998) Topical treatment of herpes simplex, herpes zoster and genital herpes with a mixture of Solanaceous glycoalkaloids. MedULA. Universidad de Los Andes. 7, 30-34.

[3] Gubarev, M.I., Enioutina, E.Y., Taylor, J.L., Visic, D.M. and Daynes, I.A. (1998) Plant-derived alkaloid protects mice against lethal infection with Salmonella typhinurium. Phyt. Res. 12, 79-88.

[4] Giron, L.M., Aguilar, G.A. Aceres, A.G. and Arroyo, G.L. (1998) Anticandidal activity of plant used for the treatment of vaginitis in Guatemala and clinical trial of Solanum nigrescences preparation. Journal Ethnopharmacology, 22, 307-313.

[5] Cham, B.E. and Wilson, L. (1987) HPLC of glycoalkaloids from Solanum sodomaeum L. Planta Medica, 53, 59-61. Doi: $10.1055 / \mathrm{s}-2006-962612$

[6] Cham, B.E., Gilliver, M. and Wilson, L. (1987) Antitumour effects of glycoalkaloids isolated from Solanum sodomaeum L. Planta Medica, 53, 34-36. Doi: 10.1055/s-2006-962612

[7] Cham, B.E. (2013) Drug therapy: Solamargine and other solasodine rhamnosyl glycosides as anticancer agents. Drug Therapy, 2(2), 33-49. Doi: 10.4236/mc.2013.22005

[8] Daunter, B. and Cham, B.E. (1990) Solasodine glycosides. In vitro preferential cytotoxicity for human cancer cells. Cancer Letters, 55, 209-220. Doi: 10.1016/0304-3835(90)90121-D

[9] Cham, B.E. and Chase, T.R. (2012) Solasodine Rhamnosyl Glycosides Cause Apoptosis in Cancer Cells. Do They Also Prime the Immune System Resulting in Long-Term Protection Against Cancer? Planta Medica, 78, 349-353. Doi: 10.1055/s0031-1298149

[10] He, D.J. (2006) Structure and anticancer activity of glycoalkaloids. Master's thesis.

[11] Lipscombe, R.J., Carter, S.J., and Ruane, M. (2005) Rhamnose binding protein. United States Patent 6, 930, 171 B2 Aug 16.

[12] Wang, Y., Gao, J., Gu, G., Li, G., Cui, C., Sun, B., and Lou, H. (2011) In situ RBL receptor visualization and its mediated anticancer activity for solasodine rhamnosides. Chemistry Biochemistry, 12(16), 2418-2420.

[13] Kuo, K.W., Hsu, S.H., Li, Y.P., Lin, W.L., Liu, L.F., Chang, L.C., Lin, C.C., Lin, C.N. and Sheu, H.M. (2000) Anticancer activity evaluation of the Solanum glycoalkaloid solamargine. Triggering apoptosis in human hepatoma cells. Biochemical Pharmacology, 60, 1865-1873. Doi:10.1016/S00062952(00)00506-2

[14] Liang, C.H., L.F. Liu, L.Y. Shiu, Y.S. Huang, L.C. Chang and K.W. Kuo, 2004. Action of solamargine on TNFs and cisplatin-resistant human lung cancer cells. Biochem. Biophys. Res. Commun., 322(3): 751-758.

[15] Kuo, K.W. and Lin, C.N. (1999) Pharmacological composition for treating cancer cells. United States Patent 6, 214-803.

[16] Cham, B.E. (2000) Medicinal Composition and their Method of Preparation. Australia Patent 779512.

[17] Cham, B.E. (2008) Cancer intralesion chemotherapy with solasodine rhamnosyl glycosides. Research Journal Biological Science, 3(9), 1008-1017.

[18] Cham, B.E. (2007) Solasodine Rhamnosyl Glycosides Specifically Bind Cancer Cell Receptors and Induce Apoptosis and Necrosis. Treatment for Skin Cancer and Hope for Internal Cancers. Research Journal Biological Sciences, 2(4), 503-514.

[19] Cham, B.E. and Meares, H.M. (1987) Glycoalkaloids from Solanum sodomaeum L. are effective in the treatment of skin cancers in man. Cancer Letters, 36, 111-118. Doi: 10.1016/0304-3835(87)90081-4

[20] Chase, T.R. (2011) Curaderm BEC5 for Skin Cancers, Is It? An Overview, Journal Cancer Therapy, 2(5), 728-745. Doi: 10.4236/jct.2011.25099

[21] Cham, B.E. (2011) Topical solasodine rhamnosyl glycosides derived from the eggplant treats large skin cancers: two case reports. International Journal Clinical Medicine, 2(4), 473-477. Doi: $10.4236 / \mathrm{ijcm} .2011 .24080$

[22] Punjabi, S., Cook, L.J., Kersey, P., Marks R. and Cerio, R. (2008) Solasodine glycoalkaloids: a novel topical therapy for basal cell carcinoma. A double-blind, randomized, placebocontrolled, parallel group, multicentre study. International Journal Dermatology, 47, 78-82. Doi:10.1111/j.13654632.2007.03363.x

[23] Goldberg, L.H., Landau, J.M., Moody, M.N., and VergilisKalner, I.J. (2011) Treatment of Bowen's disease on the penis with low concentration of a standard mixture of solasodine glycosides and liquid nitrogen. Dermatologic Surgery, 37, 858-861. Doi: 10.1111/j.1524-4725.2011.02014.x

[24] Cham, B.E. (2013) Solasodine Glycosides: A Topical Therapy for Actinic Keratosis. A Single-Blind, Randomized, PlaceboControlled, Parallel Group Study with CuradermBEC5. Journal of Cancer Therapy Vol. 2 No. 2, pp.588-596.

[25] Cham, B.E. (2013) Topical CuradermBEC5 Therapy for Periocular Nonmelanoma Skin Cancers: A Review of Clinical Outcomes. International Journal of Clinical Medicine, Vol. 4 No. 5, pp. 233-238.

[26] Cham, B.E. and Daunter, B. (1999) Glycoalkaloids. United States Patent 5958770.

[27] Solbec. (2006) Investigator's Brochure. Edition Number 6, 156.

[28] Cui, C., Wen, X., Cui, M., Goa, J., Sun, B., and Lou, H. (2012) Synthesis of solasodine glycoside derivatives and evaluation of their cytotoxic effects on human cancer cells. Drug Discoveries \& Therapeutics, 6(1), 9-17.

[29] Wu, C.H., Liang, C.H., Shiu, L.Y., Chang, L.C., Lin. T.S., Lan, C.C.E., Tsai, J.C., Wong, T.W., Wei, K.J., Lin, T.X., Chang, N.S., and Sheu, H.M. (2011) Solanum incanum extract (SRT100) induces cutaneous squamous cell carcinoma apoptosis through modulating tumour necrosis factor receptor signalling pathway. Journal Dermatological Science, 63(2), 83-92. 
[30] Shabana, M.M., Salama, M.M., Ezzat, S.M., and Ismail, L.R. (2013) In vitro and in vivo anticancer activity of the fruit peels of Solanum melongena L. against hepatocellular carcinoma. Journal Carcinogene Mutagene, 4, 149. Doi: 10.4172/21572518.1000149

[31] Cham, B.E. (2013) Inspired by Nature, Proven by Science. The new generation cancer treatment that causes cancer cells to commit suicide. $1^{\text {st }}$ Edition, Colorite Graphics, Vanuatu. ISBN=978-1-62951-158-0

[32] Cham, B.E. (In preparation) Curaderm ${ }^{\mathrm{BEC} 5}$ and Curaderm ${ }^{\mathrm{SM} 2}$ are equivalent for human basal cell carcinomas.

[33] Kantarjian, H.M., Fojo, T., Mathisen, M. and Zwelling, L.A. (2013) Cancer drugs in the United States: Justum Pretium The Just Price. Journal Clinical Oncology, 31(28) 3600-3604.

[34] CNN Money. (2010) Tanning Salons Burned by Health Care Bill. http://money.cnn.com/2010/03/24/news/economy/tanning $\operatorname{tax} /$

[35] Cham, B.E., Daunter, B. and Evans, R. (1991) Topical treatment of malignant and premalignant skin cancers by very low concentrations of a standard mixture of solasodine glycosides. Cancer Letters 59, 183-192.

[36] Cham, B.E. and Daunter, B. (1990) Topical treatment for premalignant and malignant skin cancers with Curaderm. Drugs of Today 26, 55-58.
[37] Cham, B.E. (1994) Solasodine glycosides as anti-cancer agents: Pre- Clinical and clinical studies. Asia Pacif. J. Pharmacol. 9, 113-118.

[38] Cham, B.E. (2007) Solasodine rhamnosyl glycosides in a cream formulation are effective for treating large and troublesome skin cancers. Res. J. Biol. Sci. 2(7), 749-761.

[39] Cham, B.E. (2011) Topical Solasodine Rhamnosyl Glycosides Derived From the Eggplant Treats Large Skin Cancers: Two Case Reports. Int. J. Clin. Med. 2(4), 473-477.

[40] Cham, B.E. (2012) Intralesion and Curaderm BEC topical Combination Therapies of Solasodine Rhamnosyl Glycosides Derived from the Eggplant or Devil's Apple Result in Rapid Removal of Large Skin Cancers. Methods of Treatment Compared. Int. J. Clin. Med. 3(2), 115-124.

[41] Cham, B.E. (2013) Topical Curaderm BEC5 Therapy for Periocular Nonmelanoma Skin Cancer: A Review of Clinical Outcomes. Int. J. Clin. Med. 4(5), 233-238.

[42] Cham, B.E. (2013) Drug Therapy: Solamargine and other solasodine rhamnosyl glycosides as anticancer agents. Modern Chemotherapy. 2(2), 33-49.

[43] Cham, B.E. (2014) A Review of Solasodine Rhamnosides Therapy for in-situ squamous cell carcinoma on the Penis. Brit. J. Med \& Medical Res. 4(2): 621-631. 